\title{
Comparison the Diagnostic Value of Dilatation and Curettage Versus Endometrial Biopsy by Pipelle - a Clinical Trial
}

\author{
Moradan Sanam $^{1 *}$, Mir Mohammad Khani Majid ${ }^{2}$
}

\begin{abstract}
Background: Several methods have been presented for the evaluation of the endometrium in patients with abnormal uterine bleeding, which include minimal invasive and invasive approaches such as diagnostic curettage or endometrial biopsy by Pipelle. Many studies have been performed in order to compare two methods; diagnostic curettage and outpatient endometrial biopsy. This investigation compared sampling adequacy, endometrial histopathology, failure rates, duration and costs between diagnostic curettage in a hospital and endometrial biopsy. Materials and Methods: This single blind clinical trial was performed on 130 patients older than 35 years who was referred to Amir training hospital in 2013 for elective diagnostic curettage because of abnormal uterine bleeding. For all patients eligible for the study, an endometrial sample by Pipelle was taken without anesthesia or dilatation. Then under general anesthesia diagnostic curettage was performed by sharp curette. Sampling duration was calculated and both samples were sent to the same pathologist. The diagnostic values of two methods in the diagnosis of normal endometrium, endometrial hyperplasia and carcinoma were compared. The costs of these two methods were also compared. Data analysis was performed by SPSS (version 16.0) software. Chi-Square, Fisher, and Pearson tests were used and were considered statistically significant at $P$ values less than 0.05. Results: Two methods were agreed upon $88 \%$ of sampling adequacy and $94 \%$ of pathological results. Specificity of $100 \%$ and sensitivity of $90 \%$ for detection of proliferative endometrium, secretory endometrium, simple hyperplasia without atypia and $100 \%$ for cancer were recorded. Pipelle diagnostic accuracy in comparison with curettage, have been reported over $97 \%$, so the failure rate in this study was below $5 \%$. Sensitivity of Pipelle for detection of atrophic endometrium was reported below $50 \%$. Duration and cost was lower in Pipelle versus curettage. Conclusions: It is concluded that due to high agreement and cohesion coefficient between curettage and Pipelle on the issue of sampling adequacy, histopathology finding (except atrophic endometrium), low failure rate, duration of sampling and cost, Pipelle can be introduced as a suitable alternative of diagnostic curettage.
\end{abstract}

Keywords: Abnormal uterine bleeding - biopsy of endometrium - diagnostic curettage - pipelle - endometrium sampler

Asian Pac J Cancer Prev, 16 (12), 4971-4975

\section{Introduction}

Abnormal uterine bleeding is the most common complaint among women in reproductive age attending to physicians (Fritz et al., 2011; Berek et al., 2012). Dysfunctional/anovulatory bleeding is a range of abnormal menstrual bleeding occurs in women without medical disease or pelvic disorder (Sweet et al., 2012). There are numerous methods for endometrial assessment among women with abnormal uterine bleeding including ultrasonography, endometrial curettage (D\&C), officebased methods including biopsy by hysteroscopy or endometrial samplers such as pipelle. It may help in ruling-out the endometrial hyperplasia or endometrial cancer (Fritz et al., 2011; Berek et al., 2012).

The most appropriate instrument for endometrial biopsy is pipelle (Acostin et al., 2008). Nowadays, the small flexible suction cannulas are available with less discomfort compared with old biopsy instruments but with similar results. Endometrial curettage that was previously used for evaluation of abnormal uterine bleeding is currently replaced with office endometrial biopsy. Some studies have demonstrated the sampling adequacy and good diagnostic ability of these instruments in comparison with endometrial curettage. The endometrial curettage is gold standard method of endometrial sampling. However it may lead to less than fifty percent curettage of endometrium in sixty percent of cases and is also accompanied with risk of anesthesia, infection, and perforation (Fritz et al., 2011; Berek et al., 2012). It needs hospital admission and local or general anesthesia (Coulter et al., 1993) while pipelle is an ambulatory costeffective method (Fritz et al., 2011; Berek et al., 2012). Because existing advancements and also preferred use of diagnostic curettage as the first-line diagnostic method in our center for assessment of abnormal uterine bleeding in 
women older than 35 years of age, obese younger subjects, women with long-term anovulation, abnormal uterine bleeding with endometrial thickness ranging from 5 to 12 $\mathrm{mm}$ and alone, presence of endometrial thickness more than $12 \mathrm{~mm}$, and also evaluation for endometrial cancer or hyperplasia led us to compare the diagnostic value of dilatation and curettage versus endometrial biopsy in patient with abnormal uterine bleeding to find the better method of endometrial biopsy for histopathologic evaluation with less side effects, failure ,time and costs.

\section{Materials and Methods}

A single blind clinical trial study was performed among 151 patients with abnormal uterine bleeding that were attended to Amir University hospital from September 2013 to April 2014, Semnan, Iran. The Written informed consent obtained and the study was approved by university Ethical Committee.

The exclusion criteria were focal endometrial or cervical lesion, coagulopathy, thrombocytopenia (less than $100 / 000$ platelet per $\mathrm{mm}^{3}$ ), use of anti-coagulants, pregnancy and related bleeding disorders, bleeding due to endocrinological disorders (thyroid diseases and diabetes), diseases of liver and kidney, systemic lupus erythematosus, cervical stenosis, and genital infections.

All the patients underwent transvaginal ultrasonography and who had abnormal uterine bleeding that were older than 35 years with endometrial thickness more than $12 \mathrm{~mm}$ in reproductive and premenopausal age groups and more than $5 \mathrm{~mm}$ in postmenopausal women or between 5 and $12 \mathrm{~mm}$ in reproductive and premenopausal age groups with abnormal vaginal bleeding despite medical therapy were included in the study group. After hospital admission complete blood cell count analysis, fasting blood glucose, pregnancy (BHCG), coagulative, hormonal assay consist of thyroid function test, serum prolactin, liver and kidney function tests were performed.

In this study who used less than one pad each 3 hours in bleeding period considered as normal bleeding and who used more than one pad each 3 hours, used more than 20 pads in whole bleeding periods or need to exchange the pad during sleep considered as abnormal bleeding

The patients were transferred to operation room on the scheduled day of operation and after vaginal washing and speculum insertion in lithotomy position, the sampling was performed prior anesthesia, dilatation and using of tenaculum by Pipelle (Cooper Surgical, United States). After the pipelle was inserted in the uterine cavity, the piston of the sheath was drown back to make negative gradient and then the pipelle was removed slowly. If the sample was insufficient, the procedure was repeated once or twice more. The samples were collected in container A. Then under general anesthesis dilatation and curettage was performed by using the Sims curette number 3 or 4 (monarchemical product) and tenaculum and the samples were collected in container B. The duration of sampling was calculated for both methods and was just from launch of biopsy to the end by pipelle and entering of curette to the end for endometrial curettage, also the samples were sent for histopathological evaluation by the same pathologist. The patients and pathologist were blind about the sequence of sampling and the method of sample that was used for every sample. Also the costs and time of sampling were compared across the methods. The required data were recorded by checklist.

Data analysis was performed by SPSS (version 16.0) software [Statistical Procedures for Social Sciences; Chicago, Illinois, USA]. Chi-Square, Fisher, and Pearson tests were used and were considered statistically significant at $P$ values less than 0.05 . Also the sensitivity, specificity, positive predictive value, and negative predictive value of tests were calculated.

\section{Results}

The analysis of data was performed on 130 cases and 21 cases were excluded. The mean age of the study group was $46.19 \pm 6.45$ years ranging from 37 to 57 .The mean parity was $2.9 \pm 0.89$ ranging from one to five times. Mean bleeding volume, duration and also duration of procedures were evaluated and presented on table 1 (Table 1).

Eighteen cases $(13.8 \%)$ were normal weight (BMI; 18-25), 108 subjects (83.1\%) were overweight (BMI;

Table 1. Bleeding Characteristics and Duration of Procedures

\begin{tabular}{lcccc}
\hline & Mean & Standard Deviation & Minimum & Maximum \\
\hline Bleeding duration(Day) & 2.35 & 0.567 & 1 & 3 \\
Bleeding volume(Pad/h) & 1.29 & 1.038 & 0 & 3 \\
Duration of curettage(Min) & 7.12 & 1.01 & 4 & 9.94 \\
Duration of biopsy (Min) & 3.38 & 0.98 & 1.23 & 6.29 \\
*Time duration is the exact time from initiation to the end of each procedure without consideration of time of anesthesia in D \& C
\end{tabular}

Table 2. The Frequency of Adequacy and No Adequacy

\begin{tabular}{|c|c|c|c|c|c|}
\hline & & & \multicolumn{2}{|c|}{ Pipelle Sampling adequacy } & \multirow[t]{2}{*}{ total } \\
\hline & & & yes & No & \\
\hline \multirow[t]{6}{*}{ curettage Sampling adequacy } & NO & total & 1 & 12 & 13 \\
\hline & & $\%$ Of total & $0.80 \%$ & $9.20 \%$ & $10 \%$ \\
\hline & YES & total & 109 & 8 & 117 \\
\hline & & $\%$ Of total & $83.80 \%$ & $6.20 \%$ & $90 \%$ \\
\hline & & total & 110 & 20 & 130 \\
\hline & & $\%$ Of total & $84.60 \%$ & $15.40 \%$ & $100 \%$ \\
\hline
\end{tabular}

*Yes=adequate; No=none adequat 
more than 25-30), and 4 subjects (3.1\%) were obese (BMI: more than 30).

Forty five subjects $(34.6 \%)$ had normal bleeding, 13 subjects $(10 \%)$ with pad exchange more than one pad each three hours, and 61sujects (46.9\%) more than 20 pad uses in each bleeding period, and11 subjects $(8.5 \%)$ need to exchange the pad during the night.

The endometrial thickness was less than $5 \mathrm{~mm}, 5$ to $12 \mathrm{~mm}$, and more than $12 \mathrm{~mm}$ in 12 subjects (9.2\%), 58 subjects(44.6\%), and 60 subjects (46.2\%), respectively. All those who had endometrial thickness less than $5 \mathrm{~mm}$ were postmenopausal ages.

One hundred and ten subjects $(84.6 \%)$ of the samples obtained by Pipelle and 117 subjects $(90 \%)$ of those obtained by D\& C were sufficient. The samples were sufficient in both methods in 109 subjects $(83.8 \%)$ and were insufficient in both methods in 12 subjects (9.2\%). in one subject $(0.8 \%)$ the pipelle sample had adequacy but In D\&C sample was insufficient. Eight subjects (6.2\%) had adequate sample by D\&C and were insufficient by pipelle (Table2).

The pathological results obtained by Pipelle and D \& $\mathrm{C}$ (Tables 3 ) and totally were different in 8 subjects (6.2\%).Two subject of endometrial hyperplasia without atypia, two subjects of atrophic endometrium, 3cases of proliferative endometrium and one subject of secretory endometrium were missed by pipelle. With consideration of D \& C as gold standard the sensitivity, specificity, positive predictive value, negative predictive value and accuracy are calculated and recorded (Table 4). There was more than $97 \%$ sensitivity and specificity and it means less than five percent of failure. The accordance rates of two method for diagnoses and sufficiency of samples were $94.7 \%$ and $88.4 \%$, respectively $(\mathrm{P}=0.0001)$.

The duration of biopsy was less $(3.38 \pm 0.98 \mathrm{~min})$ by pipelle method compared with D \& C $(7.12 \pm 1.01 \mathrm{~min})$ (Table 1).

The mean costs were 15.77 \$ (42120Rials) and 87.69 \$ (234200Rials) for pipelle and diagnostic curettage procedures, respectively.

Table 3. Pathological Results Obtained by Pipelle and D \& C

\begin{tabular}{lcc}
\hline Results & pipelle & D \& C \\
\hline Proliferative Endometrium & $48(36.9)$ & $51(39.2)$ \\
Secretory Endometrium & $36(27.7)$ & $37(28.5)$ \\
Simple hyperplasia without atypia & $22(16.9)$ & $24(18.5)$ \\
Cancer & $1(8)$ & $1(8)$ \\
Atrophic Endometrium & - & $2(1.5)$ \\
Undetermined & $23(17.7)$ & $15(11.5)$ \\
Total & $130(100)$ & $130(100)$ \\
\hline
\end{tabular}

\section{Discussion}

In this study $84.6 \%$ of the samples obtained by Pipelle and $90 \%$ of those obtained by D\& C were sufficient. Pipelle diagnostic accuracy in comparison with curettage, have been reported over $97 \%$, so the failure rate in this study was below 5\%. Sensitivity of Pipelle for detection of atrophic endometrium was reported below $50 \%$. The accordance rates for diagnoses and sufficiency of samples were $94.7 \%$ and $88.4 \%$, respectively.

In the study by Abdelazim et al, the pipelle and D \& C were compared (Abdelazim et al., 2013) and the authors reported $100 \%$ sufficient sample in conventional D \& C and $97.7 \%$ for pipelle that is higher by both methods in comparison to our study. It may be due to different techniques and instruments and also pathologist's experience. In a study by Naderi and colleagues (Naderi et al., 2006) the sufficiency rates were $91.6 \%$ and $98.3 \%$ by pipelle and D \& $\mathrm{C}$ respectively. These are higher sufficient rates than our study. The study by Mousavifar et al (Mousavifar et al., 2005) Reported 94\% sufficiency rate for pipelle samples that is more than results of this study. The other studies (Behnamfar et al., 2004; Fakhar et al., 2008; Bano et al., 2011) were also reported better rates for both pipelle and $\mathrm{D} \& \mathrm{C}$ in comparison with our study.Because lots of number of insufficient cases in this study were menopause and their endometrium was atrophic it is possible that the consideration of different ages groups as inclusion criteria in this and others study is the cause of differences in sufficiency rates. The other probable cause is difference in curette and pipelle that is used by different studies.

However Tanriverdi et al. (2004) Reported sufficiency rate of $88.1 \%$ and $77.1 \%$ for $\mathrm{D} \& \mathrm{C}$ and pipelle respectively that is lower than our study.

Current study showed the concordance rate of $94.7 \%$ and this rate was about $79 \%$ in Tanriverdi et al study that shows more concordance in our study (Tanriverdi et al., 2004).

Therefore, in comparison to Tanriverdi et al. (2004) study there were more sufficiency and concordance rates in this study.

In this study the diagnosis of proliferative endometrium was $36.9 \%$ and $39.2 \%$ by pipelle and D \& C methods. It was reported $26.7 \%$ by both piplle and D\&C in Abdelazim et al study (Abdelazim et al., 2013). However it was reported higher by $\mathrm{D} \& \mathrm{C}$ in our study in comparison to pipelle. The contributing rate of secretary endometrium in both was $23.5 \%$ in study by Abdelazim et al. (2013), The rates were $27.7 \%$ and $28.5 \%$ for pipelle and D \& C respectively in this study. Simple hyperplasia without

Table 4. The Accordance of Diagnoses According to the Method

\begin{tabular}{lccrr}
\hline Diagnosis & Sensitivity & Specificity & $\begin{array}{c}\text { Positive } \\
\text { predictive value }\end{array}$ & $\begin{array}{c}\text { Negative } \\
\text { predictive value }\end{array}$ \\
\hline ProliferativeEndometrium & $94.40 \%$ & $100 \%$ & $100 \%$ & $96.30 \%$ \\
Secretory Endometrium & $97.40 \%$ & $100 \%$ & $100 \%$ & $97.70 \%$ \\
Simple hyperplasia without atypia & $92.30 \%$ & $100 \%$ & $100 \%$ & $98.90 \%$ \\
Endometrial Cancer & $100 \%$ & $100 \%$ & $100 \%$ & $98.10 \%$ \\
Atrophic Endometrium & $50 \%$ & $100 \%$ & $100 \%$ & $98.50 \%$ \\
\hline
\end{tabular}


Moradan Sanam and Mir Mohammad Khani Majid

atypia was reported in $32.1 \%$ by both methods in the study of Abdelazim et al. (2013). The rates were 16.9\% and $18.5 \%$ for pipelle and D \& C respectively in our study. Despite the matter that only two patients were missed by pipelle method, regarding the importance and precancerous status of endometrial hyperplasia, it may be recommended to do D \& $\mathrm{C}$ in cases that are highly suspicious to endometrial hyperplasia or cancer.

In our study only one patient $(0.8 \%)$ had endometrial carcinoma. It was reported $7.1 \%$ by Abdelazim et al (Abdelazim et al., 2013) and there were 100\% concordance rates by $\mathrm{D} \& \mathrm{C}$ and pipelle in both studies but because there was only one case of cancer in our study the comparison between two studies is not possible.

The sensitivity, specificity, and accuracy rates were all $100 \%$ by pipelle device in our study similar to Fakhar et al. (2004) and Abdelazim et al. (2013) studies.

Also Bano et al. (2011) reported specificity of $100 \%$ and sensitivity of $93 \%$.The different rates may be due to consideration of hysterectomy as gold standard in their study. But the gold standard in our study was D \& C. Bano et al. (2011) were also reported sensitivity higher than $90 \%$ for all pathological diagnoses except atrophic endometrium and also $100 \%$ specificity rate for all diagnoses which is similar to our findings.

A study in the Spain reported $71 \%$ and $60 \%$ sensitivity for pipelle device in diagnosis of endometrial hyperplasia and cancer respectively (Antoni et al., 1997). Another study in Spain also reported sensitivity and specificity of $84.2 \%$ and $99.1 \%$ in the diagnosis of endometrial cancer and endometrial atypical hyperplasia (Machado et al., 2003) that these rates are higher in our study.

One study in United Kingdom (Sany et al., 2012) showed sensitivity rate of $96.5 \%$ and $86.5 \%$ for both $\mathrm{D} \& \mathrm{C}$ and pipelle in endometrioid and nonendometriod cancer diagnosis, but it was $100 \%$ in our study that this result probably may be due to low number of cases with cancer in this study.

In this study the sensitivity, specificity, and accuracy rates were $94.4 \%, 100 \%$, and $97.7 \%$ for diagnosis of proliferative endometrium which is lower than those reported by some studies (Abdelazim et al., 2013) and higher than some others (Fakhar et al., 2004). Also in this study the sensitivity and specificity rates were $97.4 \%$ and $100 \%$ for diagnosis of secretory endometrium. The contributing rates were $100 \%$ in previous reports (Fakhar et al., 2004; Abdelazim et al., 2013).Despite a little lower rate, the good diagnostic ability of pipelle in these cases should be remembered.

The sensitivity, specificity, and accuracy rates were $92.3 \%, 100 \%$, and $98.5 \%$ for diagnosis of simple hyperplasia without atypia in our study which is lower than $100 \%$ rate reported by other studies(Abdelazim et al., 2013; Fakhar et al., 2004) .

In Demirkiran et al. (2012) study 67\% sensitivity rate for pipelle biopsy in detection of endometrial hyperplasia was reported which is lower than our study.

Yarandi et al. (2010) reported the accuracy rates 92\% for diagnosis of endometrial hyperplasia and carcinoma by $\mathrm{D} \& \mathrm{C}$. In our study the accuracy rate were more than $98.5 \%$ and $100 \%$ for diagnosis of endometrial hyperplasia and cancer respectively.

In our study the duration of biopsy was less by pipelle device in compare to $\mathrm{D} \& \mathrm{C}$ which also was reported by Leclair et al. (2011) study.

Also in this study $83.1 \%$ of patients were overweight which shows the possible role of higher weights in pathogenesis of abnormal uterine bleeding.

The limitation of this study was doing the endometrial biopsy in operating room (for consideration of ethical issues to avoid of doing the tests in two different time and to decrease the Psychological stress of patient) just before $\mathrm{D} \& \mathrm{C}$ and if it was possible to do biopsy as outpatient it was more near to real results and May had different effect on the duration, cost or other parameters.

The strength of the study was consideration of all older than 35 years old cases that the menopause subjects also was included in the study and the diagnostic value of pipelle in evaluation of atrophic endometrium evaluated.

In conclusion, pipelle in comparison to $\mathrm{D} \& \mathrm{C}$ is a suitable device for obtaining of endometrial biopsy and would have high concordance rate for sufficiency of sample and pathological results. Also, Pipelle had high sensitivity and specificity for diagnosis of normal endometrium, simple hyperplasia without atypia and endometrial cancer. However it would have low sensitivity for diagnosis of atrophic endometrium. Regarding ability to perform the biopsy sampling by pipelle device as an ambulatory procedure and without need to anesthesia and with less duration and costs, use of this device instead of $\mathrm{D} \& \mathrm{C}$ is recommended. However it is not recommended in cases suspected to have atrophic endometrium. Also insurance coverage and also manufacturing the pipelle in internal companies would result in lower costs and more applicability.

\section{References}

Abdelazim IA, AboelezzA, AbdulKareem AF (2013). Pipelle endometrial sampling versus conventional dilatation \& curettage in patients with abnormal uterine bleeding. $J$ Turkish German Gynecol Assoc, 14, 1-5.

Agostini A, Collette E, Provansal M, et al (2008). Good practice and accuracy of office hysteroscopy and endometrial biopsy. J Gynecol Obstet Biol Reprod (Paris), 2315, 74774-4.

Antoni J, Folch E, Costa J, Foradada CM, et al (1997). Comparison of cytospat and pipelle endometrial biopsy instruments. Eur J Obsetet Gynecol Repord Biol, 72, 57-61.

Bano I, Anwar A, Tahir N, Shaheen T (2011). Establishing reliability of pipelle endometrial biopsy in comparison to traditional curettage and future outpatient hysteroscopy. Quart Med Chan, 17, 32-5.

Behnamfar F, Khamehchian T, Mazoochi T, Fahiminejad T (2004). Diagnostic value of endometrial sampling with pipelle suction curettage for identifying endometrial lesions in patients with abnormal uterine bleeding. J Res Med Sci, 3, 123-5.

Berek JS, Berek DL (2012). Berek \& Novak's Gynecology. Fifteenth edition. Philadelphia Lippincott William and Wilkins, New York pp 1-2 Numbers 374-431.

Coulter A, Klassen A, Mackenzie IZ, McPherson K (1993). Diagnostic dilatation and curettage: Is it used appropriately. BMJ, 306, 236-9.

Demirkiran F, Yavuz E, Erenel H, Bese T, arvas M, Sanioglu 
C (2012). Which is the best technique for endometrial sampling? Aspiration (pipelle) versus dilatation and curettage (D\&C). Arch Gynecol Obctet, 286, 1277-82.

Fakhar S, Saeed G, Khan AM, Alam A (2008).Validity of pipelle endometrial sampling in patients with abnormal uterine bleeding. Ann Saudi Med, 28, 188-91.

Fritz MA, Speroff L (2011). In clinical gynecologic endocrinology and infertility, $8^{\text {th }}$ ed. Lippincott Williams and Wilkins, Philadelphia PP 1-2 Numbers 604-10.

Leclair CM, Zia JK, Doom CM, Margan TK, Edelman AB (2011). Pain experienced using two different methods of endometrial biopsy: a randomized controlled trial. Obstet Gynecol, 117, 636-41.

Machado F, Moreno J, Carazo M, et al (2003). Accuracy of endometrial biopsy with the cornier pipelle for diagnosis of endometrial cancer and atypical hyperplasia. Eur J Gynaecol Oncol, 24, 279-81.

Mousavifar N, Delavari M, Talaei-Khoei M (2005). Accuracy of pipelle sampler for endometrial assessment. J Babol Univ Med Sci, 8, 53-8.

Naderi T, Asharafganjooie T, Bahrampoor A, Mehrimahani I (2006). Comparison of the diagnostic accuracy of pipelle biopsy, dilatation and curettage and hysrectomy in detection of endometrial lesions. J Kerman University Med Sci, 13, 159-63.

Sany O, Singh K, Jha S (2012). Correlation between preoperative endometrial sampling and final endometrial cancer histology. Eur J Gynaecol Oncol, 33, 142-4.

Sweet MG, Schmit Dalton TA, Weiss PM (2012). Evaluation and management of abnormal uterine bleeding in premenopausal women. American Family Physician, 85, 35-43.

Tanriverdi HA, Barut A, Gun BD, Kaya E (2004). Is pipelle biopsy really adequate for diagnosing endometrial disease. Med Sci Monit, 10, 271-4.

Yarandi F, Izadi-Mood N, Eftekhar Z, Shojaei H, Sarmadi S (2010). Diagnostic accuracy of dilatation and curettage for abnormal uterine bleeding. J Obstet Gynaecol Res, 36, 1049-52. 